\title{
Hookean atom with four electrons: On the formation of a tetrahedral Wigner molecule in the weak trapping limit
}

\author{
C. Amovilli* \\ Dipartimento di Chimica e Chimica Industriale, Università di Pisa, Via Risorgimento 35, I-56126 Pisa, Italy \\ N. H. March \\ Department of Physics, University of Antwerp, Groenenborgerlaan 171, B-2020 Antwerp, Belgium
}

(Received 23 February 2011; published 21 April 2011)

\begin{abstract}
Numerical results are presented on states ${ }^{5} S_{u}$ and ${ }^{3} P_{g}$ of the Hookean atom with four electrons. Using standard $a b$ initio quantum chemistry methods, the quintet configuration is shown to be the ground state for the weak harmonic trapping constant $k$. For large $k$ values, there is a crossover to the triplet ${ }^{3} P_{g}$ ground state. Finally, it is concluded that the Coulombic potential energy approaches the localized electron limit of a tetrahedral Wigner molecule as the harmonic force constant $k$ tends to zero.
\end{abstract}

DOI: 10.1103/PhysRevA.83.044502

PACS number(s): 31.15.A-, 31.15.V-

In earlier work, we have been concerned with the so-called Hookean model with a two-electron atom [1,2] in its ground state [3]. However, we focused on a particular force constant $k$ for the harmonic external potential

$$
V_{\mathrm{ext}}(r)=\frac{1}{2} k r^{2}=\frac{1}{2} m \omega^{2} r^{2} ;
$$

namely, $k=1 / 4$ in atomic units. For this case, in [2], the exact ground-state wave function was given analytically.

Our subsequent interest in the ground state of the Be atom [4] has prompted us to study - but now by numerical procedures to be summarized below-the Hookean model with four electrons. We were motivated to do this not only in order to understand the precise character of its ground state for a range of force constants $k$, but also to explore further our earlier interest in Wigner molecules [5,6].

In the latter context, we shall make some contact with the recent analytical study of Cioslowski and Grzebielucha [7]. However, we shall give accurate numerical results for both ground-state energies $E(k)$ and electron density $n(k ; r)$ over a wide range of trapping constants $k$, by comparing different eigenstates of $\hat{L}^{2}$ and $\hat{S}^{2}$, characterizing respectively the orbital and spin angular momentum states.

Thus, the outline of the present Brief Report is as follows. Immediately below, we shall gain orientation by first reporting $E(k)$ and $n(k ; r)$ for specific states having triplet and quintet spin character, with appropriate orbital angular momenta, by complete active space self-consistent field (CASSCF) quantum-chemical calculations. For $k \approx 1$ in atomic units, it will then become clear that the ground state in this range of harmonic trapping is a triplet. However, as $k$ is reduced, crossing of spin states will emerge, and for sufficiently small $k$, the quintet state lies lowest. With this orientation, we shall then report quantum Monte Carlo (QMC) calculations carried out for selected values of $k$. Following this we shall focus on an increasing localization of the four Coulombically repelling electrons as $k$ is greatly reduced. We shall examine,

*amovilli@dcci.unipi.it

${ }^{\dagger}$ Also at Oxford University, Oxford, England. in particular, the reduction in kinetic energy with decreasing $k$, together with the way the total potential energy $U$ is apportioned between a harmonic part $U_{H}$ and a Coulomb part $U_{C}$. The virial theorem states that the kinetic energy $T=U_{H}-(1 / 2) U_{C}$ [3]. The final part of this Brief Report is designed to show that $U_{C}$ approaches the "localized point charge" limit for a tetrahedral Wigner molecule. Also, the ground-state electron density $n(k ; r)$ is displayed in the localized Wigner regime. Finally we shall give a brief summary, supplemented by some proposals for further work that should prove fruitful.

With the above background, we shall present below a sample of diffusion quantum Monte Carlo (DMC) (and some other) results for this model atom, for a number of values of the force constant $k$ in the external harmonic potential introduced above. We shall focus on two states: namely, the ${ }^{5} S_{u}$ and ${ }^{3} P_{g}$ forms. Since we began these DMC calculations, a study has appeared by Matito et al. [8] in which configuration interaction quantum-chemical calculations were reported for the same model but for the relatively strong confinement with $k=0.01$ a.u. At this point, we also note earlier work by Varga et al. [9]. They used a form of variational quantum Monte Carlo (VMC) for a much weaker harmonic trap than in Ref. [8], corresponding to $k=0.0001$ a.u. In this regime, a lower energy was obtained for the ${ }^{5} S_{u}$ than for the ${ }^{3} P_{g}$ state, the two energies being respectively 0.3138 and 0.3159 a.u. However, they reported in another calculation, using a "shell model," that this situation reversed.

This circumstance has therefore motivated us to settle, to a prescribed numerical accuracy, the critical value of $k$, say $k_{c}$, that defines the crossover between these two states.

Table I records ground-state energies for the model atom for force constants $k$ ranging over some orders of magnitude for both of the states ${ }^{5} S_{u}$ and ${ }^{3} P_{g}$ considered throughout this Brief Report. Three types of entry occur there. First, and particularly for large $k$, which corresponds to medium or weak electron correlation, quantum chemical energies (CASSCF) are tabulated. These values were obtained with four electrons in 20 orbitals (namely $2 s, 2 p, 1 d$ and $1 f$ sets) 
TABLE I. CASSCF and quantum Monte Carlo (VMC, DMC) results for the Hookean atom with four electrons for both ${ }^{3} P_{g}$ and ${ }^{5} S_{u}$ states as a function of the trapping constant $k$ in Eq. (1). Energies are in atomic units.

\begin{tabular}{|c|c|c|c|c|c|c|c|}
\hline$\omega$ & $k=\omega^{2}$ & ${ }^{5} S_{u}(\mathrm{CAS})$ & ${ }^{5} S_{u}(\mathrm{VMC})$ & ${ }^{5} S_{u}(\mathrm{DMC})$ & ${ }^{3} P_{g}(\mathrm{CAS})$ & ${ }^{3} P_{g}(\mathrm{VMC})$ & ${ }^{3} P_{g}(\mathrm{DMC})$ \\
\hline 0.010000 & 0.0001 & 0.314520 & $0.31489(5)$ & $0.31371(1)$ & 0.315270 & $0.31721(4)$ & $0.31421(2)$ \\
\hline 0.014142 & 0.0002 & 0.404028 & $0.40432(4)$ & $0.40326(1)$ & 0.404769 & $0.40686(5)$ & $0.40391(3)$ \\
\hline 0.020000 & 0.0004 & 0.520485 & $0.52070(4)$ & $0.51975(1)$ & 0.521015 & $0.52296(6)$ & $0.52021(2)$ \\
\hline 0.024495 & 0.0006 & 0.604439 & $0.60453(3)$ & $0.60372(1)$ & 0.604660 & $0.60606(3)$ & $0.60385(1)$ \\
\hline 0.028284 & 0.0008 & 0.672540 & $0.67236(3)$ & $0.67181(1)$ & 0.672412 & $0.67372(3)$ & $0.67158(2)$ \\
\hline 0.034641 & 0.0012 & 0.782515 & $0.78227(1)$ & $0.78172(1)$ & 0.781629 & $0.78805(8)$ & $0.78102(3)$ \\
\hline 0.040000 & 0.0016 & 0.871926 & $0.87161(2)$ & $0.87106(1)$ & 0.870249 & $0.87083(7)$ & $0.86929(2)$ \\
\hline 0.500000 & 0.2500 & 6.592193 & $6.58734(1)$ & $6.58719(1)$ & 6.360332 & $6.35097(7)$ & $6.34941(2)$ \\
\hline
\end{tabular}

and all calculations were performed by using a basis set that included $5 s, 5 p, 3 d$, and $3 f$ primitive cartesian functions of gaussian type. The exponents have been optimized for any different value of the trapping constant $k$ within an even-tempered scheme. We used the GAMESS(US) package [10] for the CASSCF calculations and the CHAMP code [11] for the VMC and DMC studies. Appropriate pseudopotentials have been used in order to switch from Coulomb to harmonic external confinement. The VMC wave function has been written in a standard Slater-Jastrow form [11] with a determinantal part build by truncating the CASSCF wave function, imposing a threshold of 0.01 on the coefficients of the configuration functions. The coefficients of determinants have been reoptimized at the VMC level, together with the parameters of the Jastrow factor, using the optimization procedure implemented in the CHAMP computer program [12]. The Jastrow factor included two-body electron-nucleus and electron-electron contributions and three-body electron-electron-nucleus terms [11]. Here, the nucleus is the center of the harmonic potential. For the strongest harmonic confinement-namely, $k=$ 0.25 a.u.-recorded in Table I, these calculations clearly demonstrate that ${ }^{3} P_{g}$ is the ground state. This is also found to be the case using the DMC at a much weaker confinement corresponding to $k=0.0008$ a.u. However, VMC now makes the ${ }^{5} S_{u}$ energy lower. Then, over a range of smaller values of $k, \mathrm{QMC}$ data we have obtained are entered in Table I, for both variational and diffusion procedures.

It is relevant here to point out that there is some modest overlap between the results in Table I and the calculations reported in Refs. [8] and [9]. Where that occurs, agreement is quite satisfactory. Therefore, we stress next that, for small $k$ from 0.0004 to 0.0001 a.u., VMC and DMC both predict that the ${ }^{5} S_{u}$ form is now the ground state of this model atom. It seems safe to conclude, therefore, at the DMC level of calculation, that the critical $k_{c}$ at which the crossover occurs from ${ }^{3} P_{g}$ to ${ }^{5} S_{u}$ as $k$ decreases lies between 0.0008 and 0.0004 a.u.

Since density functional theory tells us that the ground-state energy $E=E[n]$, we show in Fig. 1 the density $n(k ; r)$ for a variety of $k$ values for the spherically symmetrical ${ }^{5} S_{u}$ state. It is clear from Fig. 1 that for weak trapping, or equivalently strong correlation, the maximum moves toward large $r$. We expect the value of $r$ at which there is a maximum to herald the onset of Wigner molecule formation and to measure the size of the tetrahedral configuration that can be expected, in the extreme localization limit, to minimize the Coulombic (C) repulsion energy between the four electrons. For the localized potential energy $U_{C}$, we know that the limit of complete

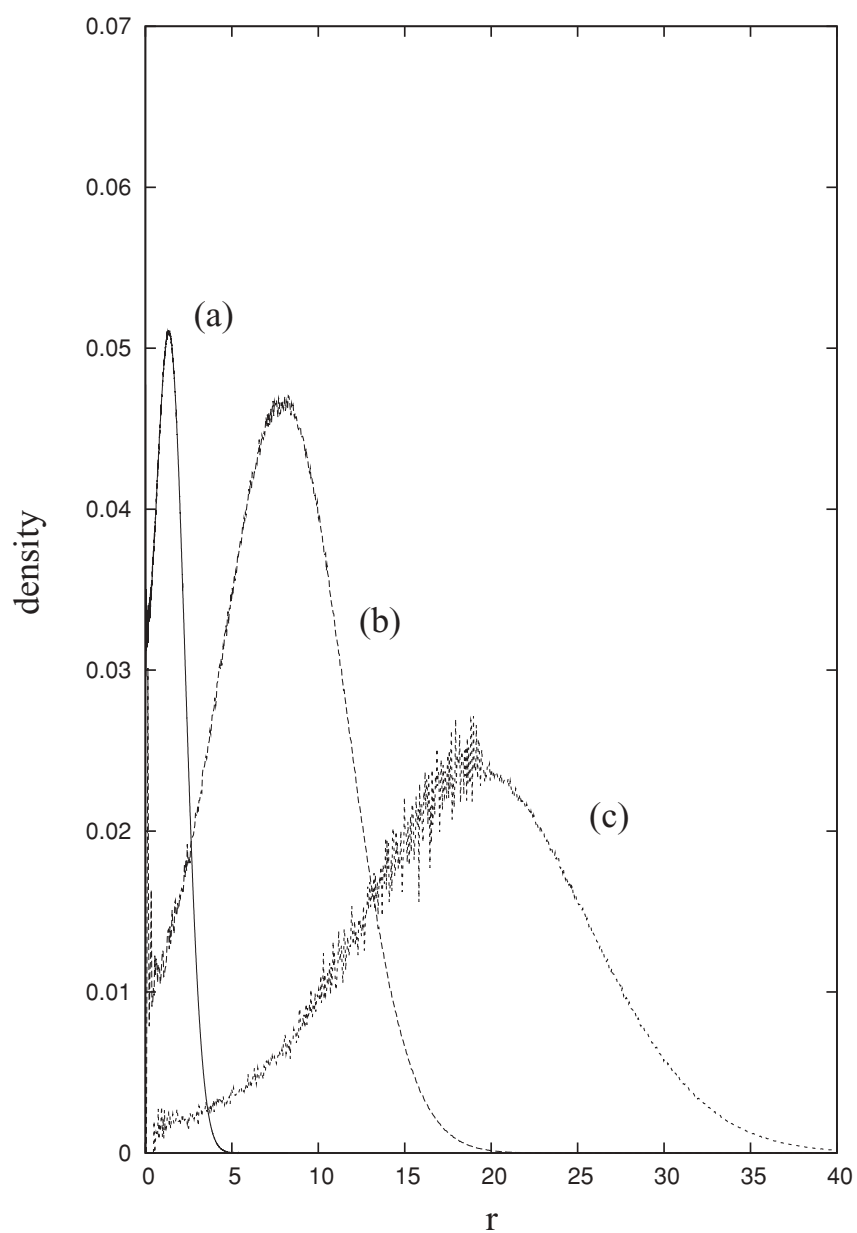

FIG. 1. Electron density $n(k ; r)$ in the Hookean atom with four electrons in the ${ }^{5} S_{u}$ state for force constants $k=0.25$ (a), $k=0.0012$ (b), and $k=0.0001$ (c) in the external potential given in Eq. (1), derived from VMC simulations. Curves (b) and (c) have been multiplied by 100 and 500, respectively, for comparison purposes. Density and length are in atomic units. 
TABLE II. Separate DMC kinetic $(T)$ and potential $(U)$ energy contributions to the total ground-state energy of ${ }^{3} P_{g}$ and ${ }^{5} S_{u}$ states. $T_{1}$ is the Jackson-Feenberg kinetic energy [14], while $T_{2}$ is the Pandharipande-Bethe kinetic energy [15].

\begin{tabular}{lcccccc}
\hline \hline$\omega$ & $k=\omega^{2}$ & ${ }^{5} S_{u}(U)$ & ${ }^{5} S_{u}\left(T_{1}\right)$ & ${ }^{5} S_{u}\left(T_{2}\right)$ & ${ }^{3} P_{g}(U)$ & ${ }^{3} P_{g}\left(T_{1}\right)$ \\
\hline 0.010000 & 0.0001 & 0.2880 & 0.0252 & 0.0257 & 0.2899 & 0.0231 \\
0.014142 & 0.0002 & 0.3661 & 0.0368 & 0.0372 & 0.3689 & 0.0340 \\
0.020000 & 0.0004 & 0.4658 & 0.0536 & 0.0539 & 0.4696 & 0.0497 \\
0.024495 & 0.0006 & 0.5364 & 0.0669 & 0.0673 & 0.5406 & 0.0618 \\
0.028284 & 0.0008 & 0.5931 & 0.0789 & 0.0787 & 0.5982 & 0.0720 \\
0.034641 & 0.0012 & 0.6833 & 0.0983 & 0.0984 & 0.6875 & 0.0951 \\
0.040000 & 0.0016 & 0.7556 & 0.1154 & 0.1154 & 0.7636 & 0.1044 \\
0.500000 & 0.2500 & 4.7473 & 1.8391 & 1.8398 & 4.7607 & 0.0506 \\
\hline \hline
\end{tabular}

localization [13] is

$$
U_{C}=\frac{3 \sqrt{6}}{2} \frac{e^{2}}{R}
$$

where $R$ is the distance from the center of the tetrahedron to each of the four vertices. Eq. (2) leads to the semiquantitative estimate of $R$ for the smallest value of $k$ considered in Table I as about 21 a.u. In relation to this point, we report in Table II the separate potential and kinetic energy contributions to the DMC total energy for the case studied in this work. The aforementioned estimate comes from $U_{C}$ evaluated from the data of Table II by means of the virial theorem-namely, by setting $U_{C}=(2 / 3)(U-T)$. This result is in some agreement with the density profile of Fig. 1.

To summarize briefly, followed by some discussion plus proposals for future directions, the major results of the present study using DMC are set out in Tables I-III and in Fig. 1. The Löwdin correlation energies [16] in Table III are worthy of some comments. First of all, it is natural enough that for the parallel spin quintet ground state, the Fermi hole precludes such electrons from coming close to one another, and we expect thereby smaller correlation energy than when antiparallel electrons are present, as in a triplet state, considered also in Tables I and III.

This prompts us to make some proposals for further work relating to the general area of this Brief Report. While the immediate connection of the Hookean atom results for four electrons with the $\mathrm{Be}$ ion sequence is not close, we anticipate more similarity with the $\mathrm{C}$ atom. Thus, the $2 s^{2}$ and the $2 p^{2}$ electrons essentially move in a Coulombic-like field with a reduced nuclear charge. In this context, we have already recorded in [17] the correlation energy of $\mathrm{Li}^{-}(-0.07217$ a.u.) relative to the $\mathrm{Be}$ atom ( -0.09434 a.u.). Of course, for Coulombic confinement $-Z e^{2} / r$ to be contrasted with Eq. (1) of the present study, one of the four electrons becomes ionized when $Z$ is reduced to about 2.9. It is tempting to view such a "phase transition" as having an analog in the Hookean model in the $k_{c}$ value, estimated with error bounds here for four electrons. Of course, no ionization is possible for harmonic confinement away from the singular point $k=0$, but the phase transition discussed here reflects a change in spin state. In the Be atom, further reduction of $Z$ below about 2.9 will eventually lead to ionization of another electron, while further transitions in Hookean confinement seem to us unlikely.

\section{ACKNOWLEDGMENTS}

N.H.M. wishes to acknowledge that part of his contribution of the present study was carried out during a visit to the University of Pisa, and he brought his contribution to fruition during a visit to ICTP (Trieste). Thanks are due V. E. Kravtsov for generously making the latter visit possible. Finally, N.H.M. thanks D. Lamoen and C. Van Alsenoy for his ongoing affiliation with the University of Antwerp through BOF-NOI.

TABLE III. Correlation energy ( $E_{\text {corr }}$, in atomic units) of a Hookean atom with four electrons for both the ${ }^{3} P_{g}$ and ${ }^{5} S_{u}$ states as a function of the trapping constant $k$ in Eq. (1). The reported correlation energies are relative to the unrestricted Hartree-Fock energy (UHF) for the ${ }^{5} S_{u}$ state and to the restricted open-shell Hartree-Fock (ROHF) energy for the ${ }^{3} P_{g}$ state.

\begin{tabular}{llcccc}
\hline \hline$\omega$ & $k=\omega^{2}$ & ${ }^{5} S_{u}(\mathrm{UHF})$ & ${ }^{5} S_{u}\left(E_{\text {corr }}\right)$ & ${ }^{3} P_{g}(\mathrm{ROHF})$ & ${ }^{3} P_{g}\left(E_{\text {corr }}\right)$ \\
\hline 0.010000 & 0.0001 & 0.326098 & $-0.01239(1)$ & 0.335397 & $-0.02119(2)$ \\
0.014142 & 0.0002 & 0.417326 & $-0.01407(1)$ & 0.428831 & $-0.02492(3)$ \\
0.020000 & 0.0004 & 0.535525 & $-0.01577(1)$ & 0.549292 & $-0.02908(2)$ \\
0.024495 & 0.0006 & 0.620508 & $-0.01679(1)$ & 0.635510 & $-0.03166(1)$ \\
0.028284 & 0.0008 & 0.689339 & $-0.01753(1)$ & 0.816990 & $-0.03354(2)$ \\
0.034641 & 0.0012 & 0.800340 & $-0.01862(1)$ & 0.907500 & $-0.03821(2)$ \\
0.040000 & 0.0016 & 0.890468 & $-0.01941(1)$ & 6.431963 & $-0.08255(2)$ \\
0.500000 & 0.2500 & 6.618917 & $-0.03174(1)$ & & \\
\hline
\end{tabular}


[1] N. R. Kestner and O. Sinanoglu, Phys. Rev. 128, 2687 (1962).

[2] S. Kais, D. R. Herschbach, N. C. Handy, C. W. Murray, and G. J. Laming, J. Chem. Phys. 99, 417 (1993).

[3] C. Amovilli and N. H. March, Phys. Rev. A 67, 022509 (2003).

[4] C. Amovilli and N. H. March, Chem. Phys. Lett. 423, 94 (2006).

[5] C. Amovilli and N. H. March, Phys. Lett. A 324, 46 (2004).

[6] P. Capuzzi, N. H. March, and M. P. Tosi, J. Phys. A 38, L439 (2005).

[7] J. Cioslowski and E. Grzebielucha, Phys. Rev. A 77, 032508 (2008).

[8] E. Matito, J. Cioslowski, and S. F. Vyboishchikov, Phys. Chem. Chem. Phys. 12, 6712 (2010).

[9] K. Varga, P. Navratil, J. Usukura, and Y. Suzuki, Phys. Rev. B 63, 205308 (2001).
[10] M. W. Schmidt et al., J. Comput. Chem. 14, 1347 (1993).

[11] C. Filippi and C. J. Umrigar, J. Chem. Phys. 105, 213 (1996).

[12] C. J. Umrigar, J. Toulouse, C. Filippi, S. Sorella, and R. G. Hennig, Phys. Rev. Lett. 98, 110201 (2007).

[13] See, for example, Eq. (28) of N. H. March, Proc. Cambridge Philos. Soc. 48, 665 (1952).

[14] H. W. Jackson and E. Freenberg, Ann. Phys. (NY) 15, 266 (1961).

[15] V. R. Pandharipande and H. A. Bethe, Phys. Rev. C 7, 1312 (1973).

[16] P.-O. Löwdin, Phys. Rev. 97, 1474 (1955).

[17] C. Amovilli, N. H. March, F. Bogár, and T. Gál, Phys. Lett. A 373, 3158 (2009). 